appear, and no epimerase is made. If the slugs are caused to differentiate into fruiting bodies they rapidly make pyrophosphorylase until its activity reaches that characteristic of fruiting bodies. If, however, the slugs already have that level of pyrophosphorylase activity before they are induced to fruit, the synthesis of a further $300-400$ units of pyrophosphorylase per $\mathrm{mg}$ of protein occurs, together with a round of synthesis of epimerase.

Newell, Longlands and Sussman (J. Mol. Biol., 58, 541 ; 1971) have now followed up these findings with a series of experiments which indicate that the regulation of synthesis of UDP-glucose pyrophosphorylase occurs at the levels of transcription and translation. After disaggregation, differentiating slime moulds reaggregate to their former stage of differentiation, but, irrespective of the amount of pyrophosphorylase present before they were disaggregated, the cells make the full quantum of this enzyme associated with differentiation. And although during undisturbed differentiation of the fruiting body synthesis of this enzyme is not inhibited by actinomycin, presumably because it involves the translation of stable preexistent messengers, its synthesis in reaggregating cells is sensitive to this antibiotic and so presumably entails further transcription. Other proteins are, however, made by reaggregating cells in the presence of actinomycin which indicates that the cells are not greatly damaged and do not loose RNAs non-specifically after disaggregation. Further, Newell and his colleagues have shown that exposing the disaggregated cells to EDTA or keeping them separated prevents synthesis of this enzyme.

During the differentiation of this slime mould the synthesis of UDP. glucose pyrophosphorylase is therefore regulated at both the transcriptional and translational levels. Cells committed to differentiation apparently transcribe the enzyme's structural gene and accumulate its mRNA which is subsequently translated when the cells receive signals telling them they have reached the appropriate stage of differentiation. After disaggregation, the cells rapidly recapitulate their morphogenetic differentiation but, as far as pyrophosphorylase synthesis goes, disaggregation seems to wipe the slate clean so that reaggregation switches on again the programmed transcription of phosphorylase mRNA which is rapidly translated. To use the words of Newell et al., the activity of UDP-glucose pyrophosphorylase and the morphogenetic changes associated with fruiting are maintained " perfectly in step". How such synchrony is established and maintained will no doubt prove to be a fascinating story.
HEALTH

Ahove the Tree line

from a Correspondent

THREE hundred delegates from thirteen countries and from the World Health Organization assembled at the University of Oulu in Finland from June 21 to 24 for the second international symposium on circumpolar health, organized by the Nordic Council for Arctic Medical Research.

The University of Oulu is the northernmost university in the world, and is 2,670 metres north of the University of Alaska, Fairbanks, where the first symposium was held in 1967. Although this claim was contested on certain technical considerations by representatives of Fairbanks in a friendly spirit, there is no doubt that the new Medical School of the University of Oulu, still partly under construction, was a perfect setting for the second symposium, with Professor Ole WaszHöckert (University of Oulu) as president.

It is an indication of the change of attitude of governments in recognizing the importance of the northern coun- tries, and of a growing awareness of the changing problems of the circumpolar peoples, that the second symposium was on a much larger scale than the first, and some two hundred contributions on a wide range of topics were presented.

On the opening day, social-economic problems and community planning and development in the Arctic and Subarctic were discussed. Although of growing political importance, the population is still very sparse. About 150,000 live today above the tree line, of whom perhaps 70,000 are "native peoples". The total population of areas north of $60^{\circ} \mathrm{N}$ lat. is 6-7 millions only, and the number of Finnish Lapps has fallen to about 3,000 . The problems of water supply, pollution and sewage disposal in conditions of perennially or seasonally frozen ground were discussed by several speakers. About 20 per cent, or 12 million square miles of the land surface of the world, is underlaid by permafrost, or ground which has been at a temperature below $0^{\circ} \mathrm{C}$ for two or more years.

On subsequent days, the symposium divided into three parallel sessions, so that delegates were able to follow their

\section{How Collagen is Made}

AN article by Lazarides and Lukens in next Wednesday's Nature New Biology buries the hypothesis that the three polypeptide chains of the collagen molecule are synthesized as one continuous chain of 300,000 molecular weight, and are then broken up into three pieces. The native collagen molecule is a three-stranded rope consisting of two types of chain, $\alpha_{1}$ and $\alpha_{2}$, differing in composition, and present in the ratio $2: 1$. Polysomes from chick embryonic tissue were fractionated on a sucrose gradient and fractions were incubated in a cell-free system to see which would produce collagen. To identify the collagen, Lazarides and Lukens took advantage of the unique feature of its composition-the presence of hydroxyproline. This amino-acid is, in fact, a product of the enzymatic modification of proline after completion of the chain. Because this cellfree system fails to modify the prolines, however, the tritium-labelled completed chains, released into the supernatant, were treated with collagen proline hydroxylase. The enzyme caused the appearance of tritiated hydroxyproline in the chain and the release of one atom of tritiated water for each proline so modified. Only the product from a heavy polysome fraction was found to be a substrate for the enzyme. The identity of the labelled product as collagen was confirmed by addition of carrier collagen chains and fractionation by gel electrophoresis, when the radioactivity followed the collagen profile.

Intact tissue was also incubated with tritium-labelled proline, and the polysomes were again fractionated by size. Completion of synthesis and release was then allowed to ensue in the presence of proline labelled with carbon-14, and the product was hydroxylated as before. The concentration of the two isotopes demonstrates comparable rates of synthesis in vitro and in vivo and also shows that there is appreciable hydroxylation in vitro while the chains are still in the process of synthesis. Adding unlabelled $\alpha_{1}$ and $\alpha_{2}$ chains, and fractionating them on an ion-exchange column, it transpires that both types are synthesized by the same polysome fraction. Their ratio, as judged by radioactivity, is anomalous, however, being $1: 1$, rather than $2: 1$, though the authors do not commit themselves about the significance of this.

From the sedimentation coefficient of the polysome fraction active in collagen synthesis, it is deduced to contain twenty-three and fifty-six ribosomes. Now a chain of 100,000 is expected to require a messenger that will accommodate thirty ribosomes. It therefore seems to follow that the collagen messenger is a monocistronic species, and that, as in all other eukaryotic systems so far studied, one messenger specifies only one polypeptide chain. 